\title{
Photoinitiator concentration and modulated photoactivation: influence on polymerization characteristics of experimental composites
}

\author{
Roberta Caroline Bruschi Alonso ${ }^{1}$, William Cunha Brandt ${ }^{2 *}$, Eduardo Jose Carvalho Souza-Junior ${ }^{3}$, \\ Regina Maria Puppin-Rontani ${ }^{3}$ and Mário Alexandre Coelho Sinhoreti ${ }^{3}$
}

\author{
* Correspondence: \\ williamcbrandt@yahoo.com.br \\ ${ }^{2} \mathrm{~S}$ chool of Dentistry, University of \\ Santo Amaro (UNISA), Prof. Eneas \\ de Siqueira Neto, 340, São Paulo, SP, \\ Brazil \\ Full list of author information is \\ available at the end of the article
}

\begin{abstract}
This study determined the influence of photoinitiator concentration on polymerization characteristics of experimental composites photoactivated by different methods. Composites based on BisGMA/TEGDMA and 65\% of filler were prepared with the concentration of camphorquinone/amine C1-0.5\%, C2-1\%, C3-1.5\%, and photocured using 4 methods: High-intensity (HI), Low-intensity (LI), Soft-start (SS) and Pulse-delay (PD). Were determined the degree of conversion (DC), depth of cure (DCure) and crosslink density (CLD). Data were submitted to ANOVA and Tukey's test $(p<0.05)$. $D C$ and DCure revealed that there were no differences among the photoactivation methods. C3 and C2 showed higher DC than C1. The CLD evaluation revealed considerable differences among the photoactivation methods. Photoinitiator concentration is a determinant factor in the polymerization characteristics of composites. Composite with low photoinitiator concentration show decreased DC and DCure, when compared with the composites with higher concentrations. Photoactivation methods had no effect on the DC and DCure. However, photoactivation methods showed differences when CLD was tested for composites with higher concentration of photoinitiators.
\end{abstract}

Keywords: Resin composites; Photoactivation; Photoinitiator; Camphorquinone; Pulse dalay; Soft-start

\section{Background}

Increasing the conversion of double bonds during the photopolymerization is critical for the optimization of mechanical properties [1,2], biocompatibility [3,4] and color stability [5] of light-activated dental resins. Photopolymerization implies both in the increase of molecular weight by monomer to polymer conversion, as well as cross-linking of developing or preexisting macromolecules [6].

In dental composites, camphorquinone (CQ) is the most employed photosensitizer or photoinitiatior. After light absorption between 400 and $500 \mathrm{~nm}$ (maximum at $470 \mathrm{~nm}$ ) $\mathrm{CQ}$ is promoted to an excited triplet state that interacts with an electron- or proton-donor molecule, like a tertiary amine, to generate free radicals $[7,8]$.

Despite of its efficiency, CQ is inherently yellow, which causes problems in color matching to natural teeth. In addition, there are also limits on the amount of amine

(c) 2014 Alonso et al.; licensee Springer. This is an Open Access article distributed under the terms of the Creative Commons Attribution License (http://creativecommons.org/licenses/by/2.0), which permits unrestricted use, distribution, and reproduction in any medium, provided the original work is properly credited. 
used by the tendency to internal discoloration of the resin. In addition to these problems, a high quantity of initiator also induces quick and high generation of free radicals, what could result in reduction of the reaction ability of the monomers due to the surrounding polymer network, and polymers with relatively lower molecular weight are formed. Also, most of the created radicals rapidly disappeared without being used in the polymerization because of their short period of life. This, in turn, places practical limits on the concentration of photoinitiators, and consequently, limits the degree of polymerization and depth of cure that can be attained [7-11].

Conversely, if the concentration of photoinitiators were kept too low, photocurable composite materials could be under-cured. If inadequate levels of conversion are achieved in the polymerization, mechanical properties and wear performance can be compromised [1], amount of leachable residual monomer increase and color stability may decline [4,5,7]. However, if conversion is maximized to reduce these difficulties, then problems inherent polymerization shrinkage of the composite become more critical [1].

Photoinitiator concentration and the light intensity can also influence polymerization rate $[8,10,11]$. The polymerization rate can regulate the cross-linking density, which is very important because a reduction in the effective cross-linking density of a cured resin will lead to a decrease in its mechanical strength, solvent resistance, and glass transition temperature [12].

Studies on alternative photoactivation methods have shown the beneficial effects of a modulated polymerization [13-15]. A slow curing allows a slow development of the composite stiffness and leads to better flow, what can reduce the shrinkage stress $[13,14]$. Previous studies $[15,16]$ have shown that the marginal adaptation of resin composites can be improved by light curing with low power density. Conversely, there are studies that claim that these benefits are consequences of lower degree of conversion [17] and that high intensity is necessary to achieve deep and complete polymerization of the material [13].

Because of this, modulated photoactivation methods as soft-start and pulse delay have been proposed [13-16]. Yoshikawa et al. [18] observed that soft-start photoactivation can lead to better marginal adaptation of composite restorations. The pulse delay photoactivation method, which is a combination of low energy density followed by a lag period before a final high power density irradiation, can also decrease the intensity of polymerization stress [13,14]. It was hypothesized that PD technique resulted in a polymer structure with lower cross-link density and that slow start polymerization techniques are associated with relatively few centers of polymer growth which may favor the formation of a more linear polymer structure with relatively few cross-links $[19,20]$. On the other hand, a rapid standard continuous cure will initiate a multitude of growth centers and a polymer with a higher crosslink density.

Considering that the aim of this study was to determine the influence of photoinitiator concentration on polymerization characteristics of experimental composites photoactivated by different methods. The first tested hypothesis was higher concentration of photoinitiator can increase degree of conversion, surface hardness, depth of cure and cross-link density. The second testes hypothesis was the photoactivation method does not affect the polymerization characteristics as degree of conversion, surface hardness and depth of cure, but the cross-link density will be higher in composites photoactivated using continuous high intensity method. 


\section{Methods}

\section{Formulation of experimental composites}

Monomer mixtures consisting of 65 wt\% of BisGMA (2,2-bis[4-(2-hydroxy-3-methacrylyloxypropoxy)phenyl]propane) and 35 wt\% of TEGDMA (triethylene glycol dimethacrylate) were prepared. The light-curing initiator system selected was camphorquinone (CQ), as photoinitiator, and dimethyl aminoethyl methacrylate (DMAEMA), as co-initiator, (proportion 1:1 by weight). The light-curing initiator system (CQ/DMAEMA) was thoroughly dissolved in the monomer matrix in the following concentrations: $0.5 \mathrm{wt} \%$ (C1), 1.0 wt\% (C2) e 1.5 wt\% (C3). Also, the inhibitor BHT (butylated hydroxytoluene) was added to the organic matrix in a concentration of $0.1 \mathrm{wt} \%$ to avoid spontaneous polymerization of the monomers. The organic matrix was reinforced with silanized barium aluminum silicate glass fillers (BaAlSi - average size: $0.5 \mu \mathrm{m})$ and silica $\left(\mathrm{SiO}_{2}\right.$ average size: $0.04 \mu \mathrm{m}$ ). The fillers were added incrementaly and mixed homogeneously up to $65 \mathrm{wt} \%$. Considering this filler content, $80 \mathrm{wt} \%$ were BaAlSi and $20 \mathrm{wt} \%$ were $\mathrm{SiO}_{2}$. The manipulation of the experimental composites was carried out under filtered orange light. The formulation of the experimental composites used in this study was selected based on the studies of Park et al. [7], Atai \& Watts [21] e Moin Jan C et al. [22]. All reagents were from Sigma-Aldrich Inc, St Louis, MO, USA.

\section{Degree of conversion}

Degree of conversion of the experimental composites was measured using FTIR spectroscopy (Bomem, model MB-102, Quebec-Canada) at a resolution of $4 \mathrm{~cm}^{-1}$ and 32 scans in the range of $4000-300 \mathrm{~cm}^{-1}$. In order to obtain the specimens $(\mathrm{n}=3)$, the experimental composites (C1, C2 and C3) were placed in a circular brass mold $6 \mathrm{~mm}$ in diameter and $2 \mathrm{~mm}$ in height and covered with polyester strips. A glass slide was placed over the strips and excess material was extruded by pressure application. The glass slide was removed and the composite was irradiated from the top through the polyester strip using the different photoactivation methods (Table 1). Subsequent to photoactivation, the specimens were finely pulverized with a hard tissue-grinding machine (Marconi, model MA590, Piracicaba-SP, Brazil) and maintained in a dark room for $24 \mathrm{~h}$. After, $10 \mathrm{mg}$ of the ground powder were mixed with $100 \mathrm{mg}$ of $\mathrm{KBr}$ powder salt. This mixture was placed into a pelleting device (Aldrich, Milwaukee, WI, USA) and then pressed in a hydraulic press (Carver Laboratory Press, model 3648, Wabash, St Morris, USA) with a load of 8 tons to obtain a pellet. This pellet was then placed into a holder attachment within the spectrophotometer (Bomem, model MB-102, Quebec-Canada) for analysis. The uncured

Table 1 Photoactivation methods used in this study

\begin{tabular}{ll}
\hline Photoactivation method* $^{*}$ & Protocol (Irradiance and Exposure time) \\
\hline Continuous high intensity (HI) & $700 \mathrm{~mW} / \mathrm{cm}^{2}$ during $40 \mathrm{~s}$ \\
Continuous low intensity $+(\mathrm{LI})$ & $150 \mathrm{~mW} / \mathrm{cm}^{2}$ during $186 \mathrm{~s}$ \\
Soft start + (SS) & $150 \mathrm{~mW} / \mathrm{cm}^{2}$ during $10 \mathrm{~s}+700 \mathrm{~mW} / \mathrm{cm}^{2}$ during $38 \mathrm{~s}$ \\
Pulse delay + (PD) & $150 \mathrm{~mW} / \mathrm{cm}^{2}$ during $10 \mathrm{~s}+3$ minutes light off $+700 \mathrm{~mW} / \mathrm{cm}^{2}$ during $38 \mathrm{~s}$
\end{tabular}

*The radiant exposure was standardized in $28 \mathrm{~J} / \mathrm{cm}^{2}$ for all groups. The light curing unit used was XL2500 (3 M/ESPE Dental Products, St. Paul, MN, USA).

tThe reduction on irradiance was obtained using a standard separators calibrated to reduced the irradiance from 700 to $150 \mathrm{~mW} / \mathrm{cm}^{2}$. 
composite resin was analyzed using a metallic siliceous window. The absorbance peaks of the cured and uncured samples of C1, C2 and C3 were obtained. The percentage of unreacted carbon-carbon double bonds $(\% \mathrm{C}=\mathrm{C})$ was determined from the ratio of absorbance intensities of aliphatic $\mathrm{C}=\mathrm{C}$ (peak at $1638 \mathrm{~cm}^{-1}$ ) against the internal reference aromatic $\mathrm{C}-\mathrm{C}$ (peak at $1608 \mathrm{~cm}^{-1}$ ) before and after curing of the specimen. The degree of conversion (DC\%) was determined by subtracting the $\% \mathrm{C}=\mathrm{C}$ from $100 \%$. Data were submitted to two-way ANOVA and means compared by Tukey's test at the $5 \%$ significance level.

\section{Cross-link density}

The degree of polymer cross-linking was assessed indirectly by measurements of the hardness before and after ethanol storage. In this evaluation, circular specimens $(6 \mathrm{~mm}$ diameter $\times 2 \mathrm{~mm}$ height) were obtained using the same circular brass mold used in the degree of conversion test. The composites $(\mathrm{C} 1, \mathrm{C} 2$, and $\mathrm{C} 3)$ were irradiated using the photoactivation methods described in Table 1. Ten specimens were obtained for each group. Immediately after light polymerization, the specimens were dark stored in air at $37^{\circ} \mathrm{C}$ for $24 \mathrm{~h}$. Next, the specimens were manually polished using 600, 1200 and 2000 grit sandpaper (Norton S.A., São Paulo, SP, Brazil) and subjected to hardness test using a Hardness Tester HMV 2000 (Shimadzu, Tokyo, Japan) at automatic procedure with a load of $50 \mathrm{~g}$ applied for $15 \mathrm{~s}$. Baseline Knoop hardness values (KHN1) were recorded. After, specimens were stored in $3 \mathrm{ml}$ of absolute ethanol solution at $37^{\circ} \mathrm{C}$ for $24 \mathrm{~h}$ and post-conditioning hardness (KHN2) was determined. Three readings were taken for each specimen and these were averaged to form a single value for that specimen. Percentage of hardness deterioration (\%HD) was computed as follows: $\% \mathrm{HD}=100-$ $(\mathrm{KHN} 2 \times 100 / \mathrm{KHN} 1)$. Data were then subjected to ANOVA and Tukey's test at the 5\% significance level.

\section{Depth of cure}

Specimens were prepared in a metallic brass containing a cavity with $3 \mathrm{~mm}$ in diameter and $5 \mathrm{~mm}$ in height, longitudinally split up in to equal parts, in order to facilitate specimen removal after polymerization. Each resin composite was inserted into the cavity in a single portion, exceeding a small amount from the margin of the cavity. The material was adapted by compression of polyester matrix under a glass slab, with static load of $1 \mathrm{~kg}$, to remove the excessive material. After the glass slab removal the specimens were light cured according Table 1. Five specimens were prepared for each composite resin (C1, C2, and C3). After light curing, the specimens were removed from the metallic mold and dark stored at $37^{\circ} \mathrm{C}$ for $24 \mathrm{~h}$ on dry environment. The specimens were fixed using wax in a vertical position in an acrylic device containing 5 cavities $(4 \mathrm{~mm} \times$ $6 \mathrm{~mm} \times 1.5 \mathrm{~mm}$ depth). Next, the specimens were ground and polished to the center, using 320, 600, 1200 and 2000 grit sandpaper (Norton S.A., São Paulo, SP, Brazil) on an automated polisher (APL-4, Arotec, Cotia, SP, Brazil). Knoop hardness values were measured across the section of the composite resin, using a Hardness Tester HMV 2000 (Shimadzu, Tokyo, Japan) at automatic procedure with a load of 50gf applied for $15 \mathrm{~s}$. Three measurement positions (A, B, and C) were made, each with five indentations (from P1 to P5) from the top to the bottom of the specimen section, giving a total of 
fifteen measurements for each specimen. The indentation layout is show in Figure 1. The values obtained in micrometers were converted to Knoop Hardness Number (KHN), by indenter software. The results were submitted to three-way ANOVA and means compared by Tukey's test at the $5 \%$ significance level.

\section{Results}

Degree of conversion values and standard deviations for all photoactivation methods are listed in Table 2.

According to two-way ANOVA, the factor composite was significant and factor photoactivation method and the interaction photoactivation method $\mathrm{x}$ composite were not significant. Considering this, in conformity with Tukey's test results, Table 2 shows that the degree of conversion was not affected by the photoactivation method, regardless the photoinitiator concentration. The concentration of photoinitiators, however, showed a significant effect. The composite with $0.5 \mathrm{wt} \%$ of CQ/DMAEMA (C1) presented significant less conversion than the others $(\mathrm{C} 2$ and $\mathrm{C} 3)$.

Indirect evaluation of cross-linking density, expressed in percentage of softening after storage in ethanol, and standard deviations for all photoactivation methods and types of composite are listed in Table 3. In this way, the higher the softening the lower the cross-linking density.

According to Table 3, cross-linking density was affected by the photoactivation method and the composite. C3 specimens polymerized with PD, SS and LI methods were significantly more susceptible to softening in ethanol than specimens cured with HI. No significant difference in cross-link density was observed between PD, SS and LI. For C2, specimens cured with LI showed the highest softening and those cured with HI the lowest softening, while specimens cured with PD and SS showed intermediate results. For C1, specimens cured with PD and LI provided significant less softening than the specimens cured with SS, while specimens cured with $\mathrm{HI}$ showed intermediate results.

Observing the photoactivation methods, when the specimens were photoactivated using SS and PD, there was no difference among the composite C1, C2 and C3: the softening was similar. For photoactivation with LI, C2 showed the highest softening, and it was significantly superior to the softening observed for $\mathrm{C} 1$ and $\mathrm{C} 3$. For photoactivation with HI, C3 showed significant lower softening than C2; C1 showed intermediated results, without significant difference from $\mathrm{C} 2$ and $\mathrm{C} 3$.

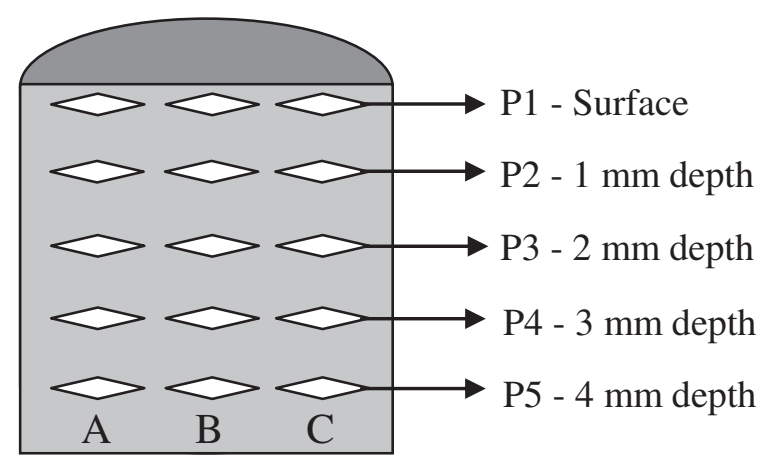

Figure 1 Schematic representation of the indentation layout. 
Table 2 Results of degree of conversion (\%) in relation to type of composite and photoactivation method, means (SD)

\begin{tabular}{ccccc}
\hline & C1 & C2 & C3 & \\
\hline HI & $47.7(1.30)$ & $50.1(0.60)$ & $54.0(3.84)$ & A \\
LI & $47.6(1.62)$ & $50.8(0.82)$ & $49.3(3.94)$ & A \\
SS & $42.0(3.41)$ & $51.8(3.45)$ & $51.8(3.74)$ & A \\
PD & $47.6(1.37)$ & $51.3(1.86)$ & $51.5(1.12)$ & A \\
& $\mathrm{b}$ & $\mathrm{a}$ & $\mathrm{a}$ & \\
\hline
\end{tabular}

Statistical differences are expressed by different capital letters in columns, and by different minuscule letters in rows $(p<0.05)$.

Depth of cure, expressed in millimeter (mm) and Knoop Hardness Number (KHN) at each depth and standard deviations for all photoactivation methods and types of composite are listed in Table 4.

In this study, the depth of cure was considered the depth at the KHN mean values had no statistical difference from those observed at the surface. The depth of cure was different for the experimental composites. The composite $\mathrm{C} 1$ showed depth of cure of $1 \mathrm{~mm}$ for all photoactivation methods while C2 showed depth of cure of $2 \mathrm{~mm}$. C3 showed depth of cure of $2 \mathrm{~mm}$ when photoactivated with LI and PD and $3 \mathrm{~mm}$ when photoactivated with $\mathrm{HI}$ and SS. Considering the KHN obtained in each depth, the hardness of the composite with lower concentration of photoinitiators $(\mathrm{C} 1)$ was significantly lowed than that observed for the other composites ( $\mathrm{C} 2$ and $\mathrm{C} 3)$ in almost all situations. In addition, it was observed that the composite $\mathrm{C} 1$ was completely unstiffened (not cured) at the depth of $4 \mathrm{~mm}$, at this depth the KHN was 0.00 for all photoactivation methods.

The superficial hardness was not affected by the photoactivation methods, regardless de composite.

\section{Discussion}

Photoinitiator concentration is a fundamental parameter that determines the polymerization characteristics of a composite $[7,8]$. This concentration is variable among the commercial composite and its effects on polymeric structure of composites is not still completely elucidated [23].

In this study the variation on concentration of photoinitiators affected all the evaluated properties, confirming the tested hypothesis. The composites with higher concentration of CQ/DMAEMA (C2 and C3) showed higher DC than the composite with lower concentration (C1). In addition, the depth of cure was also higher for $\mathrm{C} 2$ and $\mathrm{C} 3$ than for $\mathrm{C} 1$. What means that, the presence of a higher quantity of photoinitiators

Table 3 Results of indirect evaluation of cross-link density, expressed in percentage of softening after storage in ethanol (\%) in relation to type of composite and photoactivation method, means (SD)

\begin{tabular}{lccc}
\hline & C1 & C2 & C3 \\
\hline HI & $41,2(14,98) \mathrm{ABa}$ & $34,6(07,61) \mathrm{Bab}$ & $26,1(11,59) \mathrm{Bb}$ \\
LI & $37,0(08,39) \mathrm{Bb}$ & $49,8(08,50) \mathrm{Aa}$ & $41,0(11,53) \mathrm{Aab}$ \\
SS & $50,7(09,73) \mathrm{Aa}$ & $46,4(09,33) \mathrm{ABa}$ & $42,8(08,30) \mathrm{Aa}$ \\
PD & $35,6(09,28) \mathrm{Ba}$ & $44,6(11,26) \mathrm{ABa}$ & $42,8(10,76) \mathrm{Aa}$ \\
\hline
\end{tabular}

Statistical differences are expressed by different capital letters in columns, and by different minuscule letters in rows $(p<0.05)$. 
Table 4 Results of depth of cure (DCure) in relation to type of composite (C) and photoactivation method (PM), means (SD)

\begin{tabular}{|c|c|c|c|c|c|c|c|}
\hline PM & $C$ & Surface & $1 \mathrm{~mm}$ & $2 \mathrm{~mm}$ & $3 \mathrm{~mm}$ & $4 \mathrm{~mm}$ & DCur \\
\hline & $\mathrm{C} 1$ & 35.8(1.67)Ca & $37.1(3.13) \mathrm{Ca}$ & $21.5(3.61) \mathrm{Cb}$ & $02.8(0.62) \mathrm{Cc}$ & $00.0(0.00) \mathrm{Cd}$ & $1 \mathrm{~mm}$ \\
\hline \multirow[t]{3}{*}{$\mathrm{HI}$} & $C 2$ & $51.5(2.49) \mathrm{Ba}$ & $46.2(1.09) \mathrm{Ba}$ & $44.9(2.59) \mathrm{Ba}$ & 25.3(3.54)Bb & 02.9(0.22)BC & $2 \mathrm{~mm}$ \\
\hline & C3 & 56.8(3.31)Aa & 58.4(2.37) $\mathrm{Aa}$ & 52.9(1.54)Aa & 48.2(1.88)Aa & $20.4(2.50) \mathrm{Ab}$ & $3 \mathrm{~mm}$ \\
\hline & C1 & $35.0(1.66) \mathrm{Ca}$ & $34.0(2.56) \mathrm{Ca}$ & $16.0(4.50) \mathrm{Cb}$ & $02.8(1.54) \mathrm{Cc}$ & $00.0(0.00) A c$ & $1 \mathrm{~mm}$ \\
\hline \multirow[t]{3}{*}{ LI } & $C 2$ & 43.4(0.67)Ba & 43.2(5.43)Ba & 36.2(2.61)Ba & 17.4(5.97)Bb & $02.2(0.43) A c$ & $2 \mathrm{~mm}$ \\
\hline & C3 & 56.1(9.11)Aa & 53.9(4.89)Aa & 49.3(6.07)Aa & 29.5(6.59)Ab & 06.8(3.97)Ac & $2 \mathrm{~mm}$ \\
\hline & C1 & 34.1(3.67)Ca & $32.5(2.83) \mathrm{Ca}$ & $13.3(3.74) \mathrm{Cb}$ & $02.9(0.69) \mathrm{Cc}$ & $00.0(0.00) B C$ & $1 \mathrm{~mm}$ \\
\hline \multirow[t]{3}{*}{ SS } & C2 & 41.5(2.07)Ba & $40.3(2.95) \mathrm{Ba}$ & 38.8(5.23)Ba & 28.3(5.48)Bb & $06.8(2.51) A B C$ & $2 \mathrm{~mm}$ \\
\hline & C3 & 48.0(3.34)Aa & 47.4(2.34)Aa & 46.2(5.61)Aa & 40.8(3.18)Aa & 12.7(3.46)Ab & $3 \mathrm{~mm}$ \\
\hline & C1 & 33.0(2.96)Ba & 33.5(2.87)Ba & $17.8(1.88) \mathrm{Bb}$ & $03.6(1.51) \mathrm{Cc}$ & $00.0(0.00) B C$ & $1 \mathrm{~mm}$ \\
\hline \multirow[t]{2}{*}{ PD } & C2 & 47.4(6.25)Aa & 47.0(8.90)Aa & 42.6(4.61)Aa & 26.9(4.79)Bb & 10.5(8.87)AC & $2 \mathrm{~mm}$ \\
\hline & C3 & 49.1(5.12)Aa & 47.2(1.89)Aa & 47.7(2.70)Aa & 37.7(5.43)Ab & 12.8(4.00)AC & $2 \mathrm{~mm}$ \\
\hline
\end{tabular}

Statistical differences are expressed by different capital letters in columns within each group of $\mathrm{PM}$, and by different minuscule letters in rows $(p<0.05)$.

(CQ/DMAEMA) allows the generation of more radicals, which have resulted increased degree of conversion in some conditions of photocuring. Regarding the parameters analyzed, the most adequate photoinitiator concentration was $1.5 \mathrm{wt} \%$ of CQ/DMAEMA. The concentration of $0.5 \mathrm{wt} \%$ of CQ/DMAEMA was considered inappropriate for the resin matrix used.

The attempt to find an ideal concentration of photoinitiators for resin materials was the aim of a study from Moin Jan et al. [22]. Evaluating degree of conversion and photoinitiators elution, authors claimed that the better concentration of photoinitiators is $0.5 \%$ of CQ/DMAEMA in 1:1 proportion. These authors also found a decrease on the DC and increase in composite components elution (especially $\mathrm{CQ}$ ) in experimental composites with 0.8 e $0.9 \%$ de CQ/DMAEMA. At first, this result seems to be divergent to ours, since in this study the composite with $0.5 \%$ of photoinitiators showed the worst results. However, the resin monomer system evaluated in Moin Jan et al. [22] study was UDMA/TEGDMA (50:50) and no filler particles were included. In the present study, the composite has 65\% fillers and a monomer system based on BisGMA/TEGDMA (65:35). As UDMA has lower molecular weight and higher molecular mobility than BisGMA, and the quantity of TEGDMA (diluents monomer) is higher in the Moin Jan material, we could speculate that the Moin Jan material need less concentration of photoinitiators when compared to systems with higher molecular weight, as the system used in the present study. The filler particles also turn the polymerization more difficult, in this way the concentration of photoinitiators should be higher in composites than in resins.

The effect of photoinitiator concentration was also evident in the depth of cure evaluation. The depth of cure for $\mathrm{C} 1$ ( $1 \mathrm{~mm}$, regardless the photoactivation method) was lower than that observed for C2 $(2 \mathrm{~mm})$ e C3 $(2 \mathrm{~mm} / 3 \mathrm{~mm})$. In this evaluation, was also verified a significant reduction on KHN with increase of depth for all materials. It is explained by the difficult of light penetration within the composite in the deeper layers, what leads to reduction on conversion degree [24]. Therefore, reduction on degree of conversion in these areas would reduce the KHN. This reduction on KHN was more evident for $\mathrm{C} 1$ (0.5\% CQ/DAMEMA), which was completely unstiffened (not cured) at 
the depth of $4 \mathrm{~mm}$. These results corroborate with Cunha et al. [25]. Also, according to Table 3, the hardness of the $\mathrm{C} 3$ in all depths was superior or similar to $\mathrm{C} 2$, but always higher than $\mathrm{C} 1$, what also reveal the superior effectiveness of in composites with higher concentration of photoinitiators.

The results of depth of cure are supported by the results of the DC test, in which the composite with $0.5 \%$ of CQ/DMAEMA (C1) presented significant less conversion than the others ( $\mathrm{C} 2$ and $\mathrm{C} 3)$. Also, the cross-linking density is increased for composites with higher photoinitiator concentration photoactivated using high irradiance. The combination $\mathrm{C} 3$ and $\mathrm{HI}$ provided the composite with the best characteristics of polymerization.

Thus, the first tested hypothesis, that the higher concentration of photoinitiator can increase degree of conversion, surface hardness, depth of cure and crosslink density was accepted. The exception occurred when the low concentration composite (C1) was photoactivated with LI, however, it should be considered that C1 showed lower DC when compared to other composites. This difference may explain the different behavior of softening in ethanol between $\mathrm{C} 1$ and $\mathrm{C} 2 / \mathrm{C} 3$.

Another factor analysed in this study was the photoactivation method. Some studies have shown that photoactivation method can affect the polymerization characteristics of resin composites $[10,13,20]$. Modulated photoactivation methods can reduce shrinkage stress without interfere with properties such as volumetric shrinkage, degree of conversion, hardness, flexural strength, and others [13,26,27].

In this study photoactivation methods had no influence on degree of conversion, regardless the material, what corroborate whit other studies that employed different photoactivation methods with similar energy dose [13,26,27], validating part of the second tested hypothesis. Low intensity did not reduce degree of conversion because the light exposure time was increased until the polymerization reaches the diffusion limited free radical propagation [27]. It is because the polymerization is controlled by the radiant exposure. Some authors showed degree of conversion is directly related to the radiant exposure (Radiant exposure $=$ Irradiance $\times$ Light exposure) delivered to the material $[27,28]$.

The depth of cure test revealed significant difference among the photoactivation methods for $\mathrm{C} 3$ denying the hypothesis that photoactivation methods do not affect the depth of cure. Regarding this material, methods HI and SS showed depth of cure of $3 \mathrm{~mm}$ while PD and LI, $2 \mathrm{~mm}$. The likely explanation for this fact is that the long exposure time associated with the reduced irradiance in the first period of the activation increased the conversion at the superior layers and turned difficult the light diffusion within this $2 \mathrm{~mm}$ layer, reducing the conversion/KHN at $3 \mathrm{~mm}$. Conversely, for the other photoactivation methods, the initial (for HI) or quick (for SS) exposure of the material to the higher irradiance, allowed easier light incoming into the 2 first $\mathrm{mm}$ and similar conversion/KHN on the $3 \mathrm{~mm}$ depth.

Differences in network structure determine the ethanol softening, what, after all, is determined by the cross-linking density $[13,20,29]$. The exposure contact of resin composites with the solvents causes the softening of the polymeric dimethacrylate materials because attraction forces between solvent molecules and polymer chain compounds are stronger than attraction forces among the polymeric chains. The solvent penetrates within the resin matrix and expand the openings among the chains, allowing elution of residual monomers and dissolution of the linear chain $[29,30]$. The penetration ability 
of the solvent is related to its solubility parameter (ability of a molecule in penetrating and dissolving another substance). The difference in solubility parameters between polymers and solvents determines the softening of the polymer matrix: the lower the difference the higher the solvent penetration into the polymer network, the higher the softening [29,30] For composites, solubility parameter is related to formulation (monomers type/concentration) and networking (cross-linking density). More linear polymeric chains would be more degraded by the ethanol than more crosslink chains [30]. It is because, solvent may form strong secondary bond with polymer chains, replacing secondary bonds among chains (dissolution of linear polymeric network). Such polymer-solvent secondary bond, however, cannot overcome primary valence cross-link, consequently the crosslinked polymers are less soluble, reducing the softening of the network. Additionally, cross-links prevent solvent molecules from establishing adequate interactions with the polymer thus preventing the molecules to be carried off into solution [30]. In this study, all groups showed, in more or less extension, significant degradation/swelling/softening after $24 \mathrm{~h}$ ethanol storage.

The photoactivation using high intensity seems to provide a polymeric matrix more resistant. The softening in ethanol of the specimen photoactivated using HI was significantly inferior to those observed in the specimen photoactivated using LI, for all materials. For C3, $\mathrm{HI}$ also showed significant reduction on softening when compared with SS and PD. As the radiant exposure has been the same $\left(28 \mathrm{~J} / \mathrm{cm}^{2}\right)$ for all methods, this result suggests difference in cross-linking density among the methods. Possibly, the activation using continuous light in high intensity started multiple chain propagation centres, what generates higher degree of cross-linking [30]. Highly cross-linked polymers tend to be harder, inflexible, more heat-resistant and more fracture-resistant [31]. This result validates the second part of the second hypothesis.

Composites polymerized using low irradiance or using modulated photoactivation methods showed polymeric network less resistant to ethanol softening, which corroborate with previous studies, in which activation using low intensity (just initially or during the total light exposure) were associated to the fewer chain propagation centres, allowing the formation a more linear network [13,20,30]. Probably, this network presents less cross linking and more primary ciclyzation. The primary cyclization reactions create micro-gels and lead to heterogeneity in the polymer network where loosely cross-linked regions and more highly cross-linked micro-gel regions coexist. This cyclization will promote higher local conversion as it does not decrease the mobility of the system as much as crosslinking. However, cyclization can also lead to a reduction in the effective cross-linking density, since cycles do not substantially contribute to the overall network structure. According to Brandt et al. [13], irradiance, more than radiance exposure, determines the behaviour of the composite after ethanol storage.

Another aspect to discuss is depth of cure reached by specimen photoactivated using low irradiance. Cunha et al. [25] stated that the hardness not only depends on the amount of energy but also on the irradiance. Considering that, if the irradiance is reduced at the surface, it could be expected a more intense reduction on this intensity when the light passes through the composite. So, it could be expected that the method that uses low intensity (LI) would present a more accentuated reduction on KHN at the deeper areas than the other methods. However, it not occurred. As the exposure radiant was adjusted the all photoactivation methods, the polymerization depth obtained 
for all methods was quite similar. Therefore, it could be hypothesized that the regulating factor for depth of cure is the radiant exposure, and if this dose were adjusted even using a low intensity, as $150 \mathrm{~mW} / \mathrm{cm}^{2}$, the polymerization will have a pattern similar or even better than that observed for other PM.

According to the results of this study, composites with different concentration of photoinitiators show different polymerization behaviour. And, despite of the benefits of stress reduction obtained with modulated photoactivation methods or low-intensity method, it should be considered that there is a possibility of decrease on mechanical properties and low resistance to chemical degradation of the polymer matrix due to the formation of a more linear structure (low cross-linking). Thus, only the understanding of the causes of problems associated to development of techniques to reduce their consequences would help the clinicians to obtain the maximum benefits of composite restorations in clinical practice.

\section{Conclusion}

1) Photoinitiator concentration was the determinant factor on polymerization characteristics of the composites. Higher concentration of photoinitiators (C2 and C3) increased the conversion degree and the depth of cure.

2) Photoactivation methods had no effect on the degree of conversion, as the same radiant exposure was used for all methods.

3) Composites cured using modulated photoactivation methods (SS e PD) or continuous light in low intensity (LI) showed lower crosslink density when compared to composites cured using continuous light in high intensity (HI) for composite with higher concentration of photoinitiators.

Competing interests

The authors declare that they have no competing interests.

\section{Author's contributions}

RCBA carried out the depth of cure, the crosslink density, participated in the sequence alignment and drafted the manuscript. WCB carried out the manipulation of composites, analyzes the degree of conversion, participated in the sequence alignment and drafted the manuscript. EJCS-J participated in the sequence alignment and drafted the manuscript. RMP-R participated in the sequence alignment and drafted the manuscript. MACS participated in the sequence alignment and drafted the manuscript. All authors read and approved the final manuscript.

\section{Author details}

'Bandeirante University of São Paulo (UNIBAN), Rua Maria Cândida, 1813, São Paulo, SP, Brazil. ${ }^{2}$ School of Dentistry, University of Santo Amaro (UNISA), Prof. Eneas de Siqueira Neto, 340, São Paulo, SP, Brazil. ${ }^{3}$ Department of Restorative Dentistry, Piracicaba Dental School, University of Campinas (UNICAMP), Av. Limeira, 901, Piracicaba, SP, Brazil.

Received: 23 September 2013 Accepted: 19 November 2013

Published: 3 April 2014

\section{References}

1. Ferracane $J \mathrm{~L}$, Mitchem JC, Condon JR, Todd R (1997) Wear and marginal breakdown of composites with various degrees of cure. J Dent Res 76:1508-1516

2. Peutzfeldt A, Asmussen E (1996) In vitro wear, hardness, and conversion of diacetyl-containing and propanal-containing resin materials. Dent Mater 12:103-108

3. Rietschel RL (1986) Contact allergens in ultraviolet-cured acrylic resin systems. Occup Med 1:301-306

4. Nomura Y, Teshima W, Kawahara T, Tanaka N, Ishibashi H, Okazaki M, Arizono K (2006) Genotoxicity of dental resin polymerization initiators in vitro. J Mater Sci Mater Med 17:29-32

5. Imazato S, Tarumi H, Kobayashi K, Hiraguri H, Oda K, Tsuchitani Y (1995) Relationship between the degree of conversion and internal discoloration of light-activated composite. Dent Mater J 14:23-30

6. Elliott JE, Lovell LG, Bowman CN (2001) Primary cyclization in the polymerization of bis-GMA and TEGDMA: a modeling approach to understanding the cure of dental resins. Dent Mater 17:221-229

7. Brandt WC, Schneider LF, Frollini E, Correr-Sobrinho L, Sinhoreti MA (2010) Effect of different photo-initiators and light curing units on degree of conversion of composites. Brazilian Oral Res 24:263-270 
8. Pereira SG, Telo JP, Nunes TG (2008) Towards a controlled photopolymerization of dental dimethacrylate monomers: EPR studies on effects of dilution, filler loading, storage and aging. J Mater Sci Mater Med 19:3135-3144

9. Mulliken RS (1952) Molecular compounds and their spectra 2. J Am Chem Soc 74:811-824

10. Emami N, Soderholm KJ (2005) Influence of light-curing procedures and photo-initiator/co-initiator composition on the degree of conversion of light-curing resins. J Mater Sci Mater Med 16:47-52

11. Brandt WC, Tomaselli LO, Correr-Sobrinho L, Sinhoreti MA (2011) Can phenyl-propanedione influence Knoop hardness, rate of polymerization and bond strength of resin composite restorations? J Dent 39:438-447

12. Ferracane $J \mathrm{~L}$ (2006) Hygroscopic and hydrolytic effects in dental polymer networks. Dent Mater 22:211-222

13. Brandt WC, de Moraes RR, Correr-Sobrinho L, Sinhoreti MA, Consani S (2008) Effect of different photo-activation methods on push out force, hardness and cross-link density of resin composite restorations. Dent Mater 24:846-850

14. Alonso RC, Cunha LG, Correr GM, Cunha Brandt W, Correr-Sobrinho L, Sinhoreti MA (2006) Relationship between bond strength and marginal and internal adaptation of composite restorations photocured by different methods. Acta Odontol Scand 64:306-313

15. Obici AC, Sinhoreti MAC, Frollini E, Correr- Sobrinho L, de Goes MF, Henriques GEP (2006) Monomer conversion at different dental composite depths using six light-curing methods. Polym Test 25:282-288

16. Alonso RC, Correr GM, Cunha LG, De Moraes Souto Pantoja CA, Puppin-Rontani RM, Sinhoreti MA (2007) Modulated photoactivation methods-effect on marginal and internal gap formation of restorations using different restorative composites. J Biomed Mater Res B Appl Biomater 82:346-351

17. Lu H, Stansbury JW, Bowman CN (2005) Impact of curing protocol on conversion and shrinkage stress. J Dent Res 84:822-826

18. Yoshikawa T, Burrow MF, Tagami J (2001) A light curing method for improving marginal sealing and cavity wall adaptation of resin composite restorations. Dent Mater 17:359-366

19. Cunha LG, Alonso RC, Correr GM, Brandt WC, Correr-Sobrinho L, Sinhoreti MA (2008) Effect of different photoactivation methods on the bond strength of composite resin restorations by push-out test. Quintessence Int 39:243-249

20. Asmussen E, Peutzfeldt A (2001) Influence of pulse-delay curing on softening of polymer structures. J Dent Res 80:1570-1573

21. Atai M, Watts DC (2006) A new kinetic model for the photopolymerization shrinkage-strain of dental composites and resin-monomers. Dent Mater 22:785-791

22. Moin Jan C, Nomura Y, Urabe H, Okazaki M, Shintani H (2001) The relationship between leachability of polymerization initiator and degree of conversion of visible light-cured resin. J Biomed Mater Res 58:42-46

23. Taira M, Urabe H, Hirose T, Wakasa K, Yamaki M (1988) Analysis of photo-initiators in visible-light-cured dental composite resins. J Dent Res 67:24-28

24. Turssi CP, Ferracane JL, Vogel K (2005) Filler features and their effects on wear and degree of conversion of particulate dental resin composites. Biomaterials 26:4932-4937

25. Tango RN, Sinhoreti MAC, Correr AB, Schneider LFJ, Kimpara ET, Correr-Sobrinho L (2007) Knoop hardness of dental resin cements: effect of veneering material and light curing methods. Polym Test 26:268-273

26. Cunha LG, Alonso RC, Pfeifer CS, Correr-Sobrinho L, Ferracane IL, Sinhoreti MA (2007) Modulated photoactivation methods: Influence on contraction stress, degree of conversion and push-out bond strength of composite restoratives. J Dent 35:318-324

27. Halvorson RH, Erickson RL, Davidson CL (2002) Energy dependent polymerization of resin-based composite. Dent Mater 18:463-469

28. Emami N, Soderholm K, Berglund LA (2003) Effect of light power density variations on bulk curing properties of dental composites. J Dent 31:189-196

29. Schneider LF, Moraes RR, Cavalcante LM, Sinhoreti MA, Correr-Sobrinho L, Consani S (2008) Cross-link density evaluation through softening tests: effect of ethanol concentration. Dent Mater 24:199-203

30. Soh MS, Yap AU (2004) Influence of curing modes on crosslink density in polymer structures. J Dent 32:321-326

31. Gillham JK (1979) Formation and properties of network polymeric materials. Polym Eng Sci 19:676-682

doi:10.1186/2196-4351-2-10

Cite this article as: Alonso et al:: Photoinitiator concentration and modulated photoactivation: influence on

polymerization characteristics of experimental composites. Applied Adhesion Science 2014 2:10.

\section{Submit your manuscript to a SpringerOpen ${ }^{\circ}$ journal and benefit from:}

- Convenient online submission

- Rigorous peer review

- Immediate publication on acceptance

- Open access: articles freely available online

- High visibility within the field

Retaining the copyright to your article

Submit your next manuscript at $\gg$ springeropen.com 